\title{
Medicina Tradicional e Ocidental a vivência na formação do enfermeiro: relato de experiência
}

\author{
Traditional and Western Medicine experience in nursing educational background: \\ experience report
}

\section{Experiencia de Medicina Tradicional y Occidental en la formación de enfermería: informe de experiencia}

Mirelly Tavares Feitosa Pereira ${ }^{1 *}$, Felipe Alves de Almeida ${ }^{1}$, Nailu Flor Chenini de Carvalho Reis ${ }^{1}$, João Paulo Lima Barreto ${ }^{1}$, Zilmar Augusto de Souza Filho ${ }^{1}$, Noeli Neves Toledo ${ }^{1}$, Esron Soares Carvalho Rocha ${ }^{1}$, Sineide Santos de Souzaㄹ, Rizioléia Marina Pinheiro Pina ${ }^{1}$.

\section{RESUMO}

Objetivo: Descrever a experiência vivenciada pelos acadêmicos de enfermagem na disciplina Saúde das Populações Amazônicas, acerca da interação entre medicina indígena e não indígena em um Centro de Medicina Indígena em uma cidade do estado do Amazonas. Relato de experiência: A experiência foi propiciada através de uma disciplina da graduação em Enfermagem que abordou a atenção a saúde indígena e aproximou acadêmicos do conhecimento da medicina tradicional, possibilitando uma nova visão sobre o atendimento a essa população. O cenário da experiência foi o Centro de Medicina Indígena (CMI) que oferta práticas de saúde baseada na medicina tradicional indígena, além de favorecer o diálogo desta com a medicina ocidental. Considerações finais: A experiência proporcionou aos acadêmicos uma visão críticareflexiva sobre à saúde das populações indígenas, suas especificidades, complexidade do processo saúde doença, o multiculturalismo dos povos indígenas, além da formação em enfermagem capaz de favorecer um encontro intercultural enfermeiro/indígena com vistas a oferta de cuidados congruentes com a cultura e desprovido de etnocentrismo.

Palavras-chave: Enfermagem, População indígena, Ensino de enfermagem.

\begin{abstract}
Objective: To describe the experience of nursing academics in the discipline of Health of Amazonian Populations, about the interaction between indigenous and non-indigenous medicine in an Indigenous Medicine Center in a city of the state of Amazonas. Experience report: The experience was provided through a Nursing undergraduate discipline that addressed indigenous health care and brought academics closer to knowledge of traditional medicine, enabling a new vision of care for this population. The scenario of the experience was the Center for Indigenous Medicine (CMI) that offers health practices based on traditional indigenous medicine, in addition to promoting dialogue between this and western medicine. Final considerations: The experience provided a critical-reflexive view of the health of indigenous populations by the academics, their specificities, the complexity of the health care process, the multiculturalism of indigenous peoples, as well as nursing training capable of promoting an intercultural nurse/indigenous encounter with a view to offering care that is congruent with culture and devoid of ethnocentrism.
\end{abstract}

Keywords: Nursing, Indigenous population, Nursing teaching.

${ }^{1}$ Universidade Federal do Amazonas (UFAM), Manaus - AM.

*E-mail: mirelly_tavares@live.com 


\section{RESUMEN}

Objetivo: Describir la experiencia vivenciada por los estudiantes de enfermería en la asignatura salud de las poblaciones amazónicas sobre la integración entre la medicina indígena y no indígena en un centro de medicina en una ciudad del estado de amazonas. Informe de experiencia: La experiencia fue impartida a través de una disciplina de graduación en enfermería que abordó a la atención de la salud indígena y aproximó a los estudiantes al conocimiento de la medicina tradicional, posibilitando una nueva visión sobre la atención a la población. El escenario de la experiencia fue el centro de medicina indígena que aferra las prácticas de salud basada en la medicina tradicional indígena, para favorecer el diálogo de esta con la medicina occidental. Consideraciones finales: La experiencia en el ámbito de la disciplina brindó a los estudiantes una visión crítico-reflexiva sobre la salud de las poblaciones indígenas, sus especificidades, la complejidad del proceso salud-enfermedad y la multiculturalidad de los pueblos indígenas. Además, una formación que favorece el encuentro intercultural entre enfermero / indígena con el fin de brindar cuidados congruentes con la cultura y despojados de etnocentrismo.

Palabras clave: Enfermería, Población indígena, Docencia en enfermería.

\section{INTRODUÇÃO}

A atenção à saúde dos povos indígenas, histórica e culturalmente, sempre enfrentou desvalorização e descaso, sendo marcada por desafios e lutas (PINA RMP, et al., 2016). No Brasil, a Constituição de 1988 reconheceu o modo de vida dos povos indígenas, bem como sua capacidade jurídica pelos órgãos reguladores. Em 1999 foi criada a Política Nacional de Atenção à Saúde dos Povos Indígenas (PNASPI) no âmbito do Sistema Único de Saúde (SUS), a qual é operacionalizada pelos Distritos Sanitários Especiais Indígenas (DSEI) (BRASIL, 2002).

Após 21 anos, houve avanços no que se refere à extensão de cobertura e financiamento do setor, mas também limitações, como baixa efetividade e resolutividade das ações sanitárias, deficiente qualificação das equipes multiprofissionais e alta rotatividade dos profissionais (ROCHA ESC, et al., 2020). Apesar das conquistas, essas ainda são populações de contextos culturais múltiplos e singulares, que possuem sua própria forma de vivenciar a saúde e seus costumes (CASTILLO LO e DIAZ LL, 2019). Portanto, fazer saúde aplicando a legislação de forma eficiente, junto aos povos indígenas, ainda é um desafio.

Com isso, para se pensar em fazer saúde dentro do contexto indígena, é preciso analisar as ações de saúde em situação intercultural; bem como, nos responsáveis por aplicá-las, isto é, os profissionais de saúde, com destaque ao enfermeiro, por ser o gestor e maior responsável por gerenciar de forma eficaz o ambiente de diversidade étnica (ROCHA ESC, et al., 2020). Sendo assim, o desafio em fazer saúde junto aos povos indígenas é traduzido pela necessidade de reconhecimento da diversidade de cada povo e pela garantia de acesso equitativo aos serviços com qualidade por parte do profissional enfermeiro (ROCHA ESC, et al., 2020). Portanto, para se aplicar o fazer saúde aos indígenas com qualidade e de maneira efetiva é preciso refletir acerca de como os enfermeiros têm sido preparados para lidar com as múltiplas realidades e gerenciar a saúde em contexto intercultural (CASTILLO LO e DIAZ LL, 2019).

Dessa forma, a inserção da temática saúde indígena deve ter origem ainda na graduação, possibilitando aos futuros profissionais de enfermagem o desenvolvimento de competências e habilidades que permitam lidar com os desafios, tais como, liderança, tomada de decisão, pensamento crítico-reflexivo sobre as ações (ROCHA ESC, et al., 2020). Ou seja, é necessário que a formação destes profissionais possua direcionamento para lidar em situações de interculturalidade, atendendo às necessidades sociais e epidemiológicas do contexto multicultural.

Ressalta-se ainda que o manejo da diversidade no fazer saúde de forma equitativa difere de acordo com os cenários brasileiros, por suas dimensões continentais e múltiplas culturas. Por isso, na região Norte a observação do contexto indígena é mais proeminente, por ser a região que mais engloba povos indígenas (BRASIL, 2019). Cabe destacar que no Amazonas concentra-se aproximadamente $20,6 \%$ da população brasileira autodeclarada indígena que reside dentro e fora de Terras Indígenas demarcadas (IBGE, 2012). 
Portanto, pensar na aplicação das políticas públicas no que tange aos povos indígenas, se faz mais necessário nesta região; sendo também essa inferência verdadeira no que concerne à aplicação do Subsistema de Atenção à Saúde Indígena (SASI), da PNASPI e na formação do profissional enfermeiro com capacidades interculturais (PEREIRA PPG, 2012; BRASIL, 2019).

Diante do exposto, esse relato tem como objetivo descrever a experiência de acadêmicos de enfermagem na disciplina Saúde das Populações Amazônicas, acerca da interação entre medicina indígena e não indígena em um Centro de Medicina Indígena em uma cidade do estado do Amazonas.

\section{RELATO DE EXPERIÊNCIA}

Trata-se de um estudo descritivo, tipo relato de experiência por meio da sistematização de experiências na perspectiva de Holliday OJ (2002). A disciplina proporcionou prática no âmbito da saúde indígena, o que aproximou os acadêmicos à realidade dessa população. A experiência teve como cenário um Centro de Medicina Indígena (CMI) que oferta práticas de saúde baseada na medicina tradicional indígena, além de favorecer o diálogo desta com a medicina ocidental.

O CMI foi fundado em 2017 em Manaus e foi idealizado para oferecer tratamentos de saúde com técnicas terapêuticas indígenas. A criação foi pensada a partir do conflito existente entre a medicina ocidental e a medicina tradicional indígena, como parte da luta pela política de atendimento à saúde diferenciada, com a intenção de promover um espaço de diálogo e articulação entre outros sistemas de saúde, sendo uma proposta de fortalecimento da identidade étnica, cultural e a organização social dos povos originários além de contribuir como fonte de renda para as famílias e comunidades indígenas.

O CMI oferta a medicina tradicional indígena, cursos de línguas indígenas, cosmologias indígenas e saúde, além de produção e venda de artesanato e medicamentos à base de plantas. Além disso, o espaço não se restringe a consultas e tratamentos de saúde com terapêuticas indígenas, é também um espaço incentivador de surgimento de novos CMI's em outras regiões. Logo, envolve lideranças em nível local, regional e nacional provocando debates sobre os saberes indígenas e sua urgência para reconhecimento de fato e de direito.

A experiência relatada foi vivenciada por acadêmicos do oitavo período do curso de enfermagem, durante a disciplina de Saúde das Populações Amazônicas de uma Instituição de Ensino Superior (IES) pública do Amazonas. A disciplina objetiva proporcionar uma visão holística dos aspectos socioculturais e de saúde relacionado às populações amazônicas com um olhar atento às populações indígenas, por considerar expressiva essa população na região Norte, especificamente no Amazonas.

Realizou-se a coleta de dados a partir da proposta metodológica de Holliday OJ (2002), a qual é realizada em cinco etapas: 1) O ponto de partida: relata a vivência das experiências, portanto, é o início do processo de sistematização, no qual foi necessário recorrer aos registros e anotações a respeito das experiências vivenciadas pelos discentes de enfermagem; 2) As perguntas iniciais: definição e delimitação dos objetivos a sistematizar, ou seja, identificação do eixo da sistematização da experiência surgiu então, a partir da experiência de uma exposição dialogada mediada por um indígena, que apresentou os modos de vida, crenças e cosmovisão dos povos indígenas no contexto da sua etnia, além da visita guiada ao CMI durante o segundo semestre de 2019. 3) Recuperação do processo vivido: é a reconstrução da história, onde as informações foram ordenadas, classificadas e descritas conforme os acontecimentos; 4) A reflexão de fundo: nesse momento foi necessário analisar, interpretar e sintetizar a experiência através de embasamento teóricocrítico e reflexivo; 5) Os pontos de chegada: etapa final, na qual foram formuladas as conclusões desta experiência, evidenciando uma experiência transformadora.

O resgate das informações foi por anotações realizadas em sala de aula com participação de um indígena convidado para contribuir na disciplina, além da visita ao CMI, que serviram de embasamento para a construção da experiência transformadora. Diante disso, o ponto de partida deu-se por meio de diálogos com o indígena que proporcionou aos acadêmicos o conhecimento sobre a realidade do contexto e serviço de saúde indígena, despertando interesse e motivando a busca por compreensão sobre o processo saúdedoença, itinerário terapêutico e medicina tradicional indígena e sua articulação com a medicina ocidental. 
As perguntas iniciais surgiram a partir das vivências relatadas no primeiro ponto. Ao qual destacamos: "Qual a importância de uma disciplina que aborda saúde indígena para os acadêmicos de enfermagem?"; "Qual a relevância do contato direto com os indígenas e com a medicina tradicional/práticas terapêuticas utilizadas por eles, e como esse contato observacional agrega na formação do acadêmico de enfermagem?". A partir destes questionamentos foi possível delimitar os objetivos deste relato e refletir sobre a relevância do contato direto com os indígenas, conhecer suas práticas terapêuticas e a inclusão do processo de cuidar seguindo o conceito da equidade na formação do futuro profissional de enfermagem, sendo essa inclusão $o$ principal eixo da discussão.

A disciplina tem como objetivo proporcionar aos acadêmicos uma visão holística sobre os aspectos socioculturais relacionados ao processo saúde-doença das populações amazônicas. Durante a disciplina houve a participação de um indígena da etnia Tukano para dialogar com os acadêmicos sobre a visão indígena do processo saúde-doença, como veem a medicina ocidental e até que ponto ela é utilizada por eles, bem como a visão que possuem sobre a articulação entre medicina tradicional indígena e medicina ocidental.

A proximidade com o indígena, o diálogo e a escuta a respeito da medicina tradicional indígena, aliado à visita ao $\mathrm{CMI}$ foram estratégias de ensino que favoreceram uma aprendizagem significativa, pois ao desvelar a visão de mundo do indígena, percebeu-se que as práticas de saúde devem ser ressignificadas com vistas a contemplar, em sua totalidade as demandas de saúde dessa população.

O CMI localiza-se no centro histórico da capital amazonense, no espaço são oferecidas práticas de saúde e produtos naturais para a prevenção de doenças e resolução de problemas de saúde. O serviço é destinado à população indígena e não indígena que busque práticas terapêuticas indígenas. Como práticas de saúde principais o CMI utiliza-se de ervas e "bahsese", também denominado de "benzimento", realizado por um pajé. A imersão nesses ambientes promoveu aos acadêmicos conhecimento significativo sobre outras formas de cuidar e de tratamentos utilizados por outros povos, o que permitiu a compreensão de outros pontos de vista e o desenvolvimento do pensamento crítico-reflexivo.

\section{DISCUSSÃO}

O contato com outras culturas e tratamento pedagógico de relações étnico-raciais ainda é uma abordagem simplista nos currículos das IES, isto é, ainda é uma temática superficial na educação e causa defasagem na formação de profissionais que lidam com tais questões ao longo de sua carreira, como é o caso da enfermagem (MENERZ CB, 2017). Sendo assim, práticas diretas interculturais contribuem para uma formação crítica-reflexiva com amplitude transformadora sobre a realidade. Portanto, o contato direto com o indígena durante o diálogo em sala e a visita ao CMI promoveu significados singulares no âmbito da formação acadêmica, visto que possibilitou aproximação com outra realidade cultural e permitiu a harmonização entre teoria e prática, contribuindo para a consolidação do conhecimento necessário para o cuidado intercultural e equânime.

Estudos revelam que a academia tem falhado em gerar profissionais preparados para lidar com as diversas particularidades étnicas, principalmente no Brasil, haja vista sua riqueza cultural, inclusive indígena (NASCIMENTO VF, et al., 2020). Logo, ao sair da academia o profissional aplica de forma impositiva o que foi aprendido ao longo de sua formação sobre o processo saúde-doença, e por vezes, desrespeita as peculiaridades culturais, que são imprescindíveis para um cuidado de saúde eficaz (FERRAZ L, BOEHS AE, FERNANDES GCM, 2013; CASTRO NJC, 2015; FONTANA RT, 2019).

Observa-se a necessidade de avanços na formação do enfermeiro para atuar em contextos interculturais. Estudo realizado com enfermeiros que objetivou identificar dificuldades encontradas na assistência à população indígena, evidenciou, entre as dificuldades, falta de treinamento ao iniciar o trabalho e pouca aceitação do profissional por parte do indígena, demonstrando necessidade de capacitação para o trabalho intercultural e qualificação continuada. Essa ausência de capacitação ocasiona sentimento de insegurança quanto à assistência ao indígena e afeta o cuidado efetivo (MARINELLI NP, et al., 2012). 
Pereira PPG (2012), em estudo com profissionais de saúde que atuam em contextos indígenas, traz a reflexão sobre os desafios enfrentados por estes ao se depararem com concepções diferenciadas de corpo, saúde, doença, práticas de saúde e como os profissionais são afetados por essa diversidade. Refere que o encontro de profissionais com os indígenas e sua visão de mundo pode afetar e modificar a prática profissional por meio da compreensão de que a biomedicina não é a resposta para todos os problemas de saúde e possui limitações, sendo necessário reconhecer os limites do próprio conhecimento e estar aberto para outros saberes.

As aulas foram conduzidas de forma a favorecer a reflexão sobre o cuidado diferenciado ao indígena, pois proporcionou contato in loco com ambientes e indivíduos que vivenciam a saúde de populações etnicamente diferentes. Louzada J e Neto DL (2010) ressaltam que o trabalho em área indígena envolve vários desafios, como o deslocamento do profissional para a aldeia, a inserção num mundo desconhecido na maioria das vezes, além do estabelecimento de vínculo profissional-indígena, o que requer capacitação antropológica.

A disciplina ofereceu experiências significativas por favorecer a apreensão sobre as discussões durante as aulas. O futuro profissional enfermeiro deve se despojar dos preconceitos com vistas a promover um cuidado individualizado considerando as especificidades étnicas (CASTILLO LO e DÍAZ LL, 2019).

$O$ estudo sobre outros povos e culturas favorece o conhecimento sobre as particularidades e singularidades existentes (CORDEIRO GOG, et al., 2020). É preciso avançar nas discussões sobre transculturação, espiritualidade, saúde indígena e Política de Saúde da População Negra pelas suas peculiaridades para que os enfermeiros possam compreender as diferenças culturais dos grupos étnicos, os significados, as implicações e a subjetividade existente no processo de cuidado (LIMA MRA, et al., 2016).

Sendo assim, estudar saúde indígena com o contato direto com indígenas permitiu conhecer diversas culturas, etnias e práticas terapêuticas da medicina tradicional indígena. A vivência por meio da aproximação com a realidade da saúde indígena, aliadas ao conhecimento científico, pode favorecer aos futuros enfermeiros a oferta de cuidados diferenciados, eficazes e equânimes a essa população, haja vista que competências culturais estão sendo trabalhadas em sua formação (CUERVO MRM, et al., 2015).

A experiência dos acadêmicos na disciplina Saúde das Populações Indígenas foi importante para a formação em enfermagem por apresentar diferentes formas de cuidados de saúde em diferentes culturas, que a medicina ocidental precisa reconhecer. As atividades desenvolvidas na disciplina provocaram transformações positivas na formação, reflexões sobre a importância de considerar a multiculturalidade no planejamento dos cuidados aos povos em questão, além de sensibilizar os acadêmicos sobre outras formas de cuidados existentes que precisam ser respeitados, bem como a necessidade de realizar a escuta sensível, valorizando as particularidades inerentes às questões culturais para oferecer cuidado holístico, efetivo, e assim, gerar impactos positivos na atenção à saúde das populações.

A experiência descrita foi importante ao aprendizado significativo à medida que apresentou aproximação com a cultura indígena, a cosmovisão e o processo saúde-doença vivenciados pelos indígenas e por favorecer a compreensão de que para cuidar do indígena de forma integral e equânime é necessário desconstruir conceitos pré-concebidos e visões equivocadas e etnocêntricas.

A disciplina favoreceu a construção de novos conceitos sobre o cuidado, sensibilizou os futuros profissionais para a necessidade de um olhar mais amplo às demandas da população indígena e sensível para atuar de maneira eficaz no contexto da saúde indígena e promoveu a aquisição de competências culturais para tornar o cuidado holístico e humanizado, que respeita as crenças e costumes. Dessa forma, o indígena desfrutará de uma assistência segura, respeitosa e isenta de etnocentrismo.

\section{AGRADECIMENTOS}

Agradecimentos a Escola de Enfermagem de Manaus da Universidade Federal do Amazonas, aos docentes da disciplina Saúde das Populações Amazônicas e ao Centro de Medicina Tradicional Indígena pela acolhida, diálogo intercultural e construção de novos conhecimentos. 


\section{REFERÊNCIAS}

1. BRASIL. Fundação Nacional de Saúde. Política Nacional de Atenção à Saúde dos Povos Indígenas. Brasília: Ministério da Saúde, 2002.

2. BRASIL. Ministério da Saúde. Saúde Indígena: Análise da situação de saúde no SASISUS. Secretaria Especial de Saúde Indígena. Brasília: Ministério da Saúde. 2019.

3. CASTILLO LO, DÍAZ LL. Percepción del cuidado de enfermería por indígenas Embera.: Perception of nursing care by Embera Indigenous people. Rev. cienc. cuidad. 2019. Apr. 16(2):72-82.

4. CASTRO NJC, et al. Ensino da Saúde das Populações Tradicionais em Cursos de Enfermagem. Enferm. em foco. 2019. 10 (6): 36-41.

5. CASTRO NJC. O ensino da saúde indígena nos currículos e espaços acadêmicos. Rev. ensino saúde ambien. 2015. 8(1):15-25.

6. CORDEIRO GOG, et al. Hitupmã'ax: educação intercultural e atendimento diferenciado à saúde do povo maxakali. Hist. cienc. saúde-Manguinhos [Internet]. 2020 Mar; 27(1): 199-218

7. CUERVO MRM, et al. PET-Redes de atenção à saúde indígena: além dos muros da universidade, uma reflexão sobre saberes e práticas em saúde. Interface (Botucatu) [internet]. 2015. 19 Suppl 1:953-63.

8. FERRAZ L, BOEHS AE, FERNANDES GCM. Percepções das Equipes de Saúde da Família sobre cuidado profissional às crianças indígenas. Cienc Cuid Saúde. jan./mar; 12(1):032-039, 2013.

9. FONTANA RT. A interculturalidade na formação dos profissionais de enfermagem. Rev. contexto educ. [periódico na Internet]. 2019. 34(109):36-51.

10. HOLLIDAY OJ. Para sistematizar experiências. $2^{2}$ ed. revista - Brasília: MMA; 2006. p. 71 - 92.

11. IBGE. Os indígenas no censo demográfico de 2010: primeiras considerações com base no quesito cor ou raça. Instituto Brasileiro de Geografia e Estatística. Brasília: Ministério do Planejamento, Orçamento e Gestão, 2012.

12. LANDGRAF J, et al. Desafios para a Educação Permanente em Saúde Indígena: adequando o atendimento do Sistema Único de Saúde no sul do Brasil. Interface (Botucatu). 2019. 24:190166.

13. LIMA MRA, Nunes MLA, Klüppel BLP, Medeiros SM, Sá LD. Nurses' performance on indigenous and African-Brazilian health care practices. Rev Bras Enferm [Internet]. 2016;69(5):788-94.

14. LOUZADA J, NETO DL. Abordagem crítico-interpretativa das fragilidades e potencialidades do trabalho de enfermagem aos ianomâmis, Amazonas. Enferm. em foco [Internet]. 2010. 1(2): 42-5.

15. MARINELLI NT, Nascimento DF, Costa AIP, Posso MBS, Araújo LP. Assistência à população indígena: dificuldades encontradas por enfermeiros. Rev Univap. 2012; 18 (32).

16. MENERZ CB. Ensino de história, diálogo, intercultural e relações étnico-raciais. Educ. Real [periódico na internet]. 2017. 42(1):59-77.

17. NASCIMENTO VF, et al. Desafios na formação de enfermeiros indígenas em Mato Grosso, Brasil. Ciênc. Saúde Coletiva. 2020. 25(1):47-56.

18. PEREIRA PPG. Limites, traduções e afetos: profissionais de saúde em contextos indígenas. Mana 2012; 18:511-38.

19. PINA RMP, et al. Ensino de enfermagem na saúde indígena: uma abordagem problematizadora - Relato de Experiência. Journal of Nursing UFPE online. 2016. 10 Supl.3:1556-61.

20. ROCHA ESC, et al. Atuação da enfermagem na atenção à saúde indígena no contexto brasileiro. In: Associação Brasileira de Enfermagem; Kalinowski CE, Crozeta K, Costa MFBNA, organizadoras. PROENF Programa de Atualização em Enfermagem; Atenção Primária e Saúde da Família: Ciclo 8. Porto Alegre: Artmed Panamericana: 2020. P. 109-28.

21. ROCHA ESC, et al. Profile of nurses who work in indigenous and non-indigenous health care. Cienc. Cuid. Saúde. 2019; 17(4). 